\title{
The use of robotic or laparoscopic stapler in rectal cancer surgery: a systematic review and meta-analysis
}

\author{
P. Tejedor ${ }^{1}$, F. Sagias ${ }^{1}$, K. Flashman ${ }^{1}$, N Li Kandala ${ }^{2}$, JS Khan ${ }^{1}$. \\ 1. Department of Colorectal Surgery, Queen Alexandra Hospital, Portsmouth, UK. \\ 2. Faculty of Sciences, School of Health Sciences \& Social Work, University of Portsmouth, \\ UK.
}

\section{Correspondence to:}

Jim S Khan.

Queen Alexandra Hospital, Portsmouth, UK.

Southwick Hill road Cosham, Portsmouth, PO6 3LY, UK

Tel 02392286000

Email: jim.khan@ porthosp.nhs.uk

Article type: Review article and meta -analysis

Word count, body text: 1446 words

Word count, abstract: 275 words

Figures: 3

Tables: 1

Disclosures: None

\section{AUTHOR'S CONTRIBUTION}

All authors critically revised the paper for important intellectual content. All authors have contributed to the work and agreed on the final version. This manuscript is not being considered by any other journal.

\section{KEY WORDS:}

Robotic stapler; Anastomotic leak; Total mesorectal excision; Rectal cancer; stapler firings; meta-analysis. 


\section{ABSTRACT}

Purpose

Minimally invasive surgery for total mesorectal excision (TME) remains technically challenging due to poor manoeuvrability within the pelvis, which makes extremely difficult to introduce a laparoscopic stapler (LS) for the rectal transection. We aim to perform a systematic review and meta-analysis on robotic TME after the use of robotic stapler (RS) or LS after robotic TME.

\section{Method}

A systematic literature search was performed using PubMed, MEDLINE and Cochrane Database. Participants who underwent robotic anterior resection were considered following these criteria: 1) studies comparing RS and LS; 2) studies reporting the rate of anastomotic leakage (AL). The primary outcome was the risk of AL. Secondary outcomes included the number of firings of stapler needed.

A meta-analysis based on Preferred Reporting Items for Systematic Reviews and Meta-analyses (PRISMA) statement was performed.

\section{Results}

A total of 4 studies including 391 patients $(L S=251, \mathrm{RS}=140)$ were included for analysis. Regarding the number of firings, overall results suggest that the rectal stapling was achieved with less firings in the RS group, but the difference is not significant [risk difference, $(95 \% \mathrm{CI}): 0.42(-0.13,0.97)]$. There was a $56 \%$ increased odds of AL for LS compared with RS; however, this was not statistically significant (OR: 1.56; 95\% CI: 0.59, 4.14)

\section{Conclusion}

With the current literature evidence, the present study could not demonstrate a clear benefit of the RS over the LS. Although the development of an AL is multifactorial and unpredictable, our data may suggest that the use of the RS could help reducing the risk of AL. There is a need of future randomized clinical trial in order to assess the possible benefits of the RS. 


\section{INTRODUCTION}

Minimally invasive surgery for total mesorectal excision (TME) remains technically challenging, mostly because of poor manoeuvrability within the pelvis. A narrow pelvis and the unfavourable entry angle make it extremely difficult to introduce a laparoscopic linear stapler for the rectal transection. The exposure can be a problem and finally stapling of the rectum can be challenging.[1] Published data supports that three or more firings significantly increase the rate of AL (hazard ratio 7.8, 95\% confidence (CI) 3.8-16.3),[2,3] although the development of an anastomotic leak (AL) is multifactorial, including several variables such as male sex, previous radiotherapy in the pelvis, bowel preparation, height of the anastomosis and other comorbidities.[4,5]

Stapling in the pelvis remains an Achilles heel of laparoscopic rectal surgery and, therefore, one of the most practical innovations of the robotic system is the use of the robotic stapler (EndoWrist Stapler, Intuitive Surgical, Inc.). It has a wide range of side-to-side and up-and-down articulation, enabling a more accurate positioning.

With this systematic review and meta-analysis, we aimed to provide a comprehensive overview of the current data regarding anastomotic leak and number of firings with the use of a robotic stapler.

\section{METHODS}

\section{Information sources and search}

We performed a systematic literature search in accordance with Preferred Reporting Items for Systematic Reviews and Meta-analysis (PRISMA) statement. A comprehensive search of published studies comparing LS with RS in rectal cancer surgery was performed in the following databases: PubMed, MEDLINE and Cochrane Database of Systematic Reviews. The primary search strategy included keywords as follows: 'Robotic stapler; Robotic rectal surgery; stapler firings'. The process selection is represented in Figure 1.

\section{Inclusion and exclusion criteria}

According to the PICOS criteria, studies were selected in this meta-analysis according to the following eligibility criteria: 1) Participants: Patients diagnosed with rectal cancer; 2) 
Intervention: robotic total mesorectal excision for rectal cancer; 3) Comparison: between robotic stapler and laparoscopic stapler; 4) Outcomes: intraoperative details and postoperative anastomotic leakage rate compared between two groups; 5) Study design: cohort studies or matched case-control trials.

The exclusion criteria were: 1) no robotic stapler group; 2) absence of the outcome of interest.

\section{Endpoints and data extraction}

The primary endpoint was to compare the differences between the RS and LS groups regarding anastomotic leakage. Secondary outcomes included intraoperative data, such as number of firings needed.

Two authors (PT and FS) independently screened the titles and the abstracts of all identified studies and excluded those deemed irrelevant. Full text articles of potentially relevant studies were obtained. These studies were assessed independently by the two authors (PT and FS) as to whether they met the inclusion criteria for this review, and the results were confirmed by the senior author (JSK).

\section{Statistical analysis}

The meta-analysis was performed using RevMan software version 5.1. To compare the rate of anastomotic leakage, odds ratios (OR) with $95 \% \mathrm{CI}$ were used as a measure of efficacy. Continuous variables representing the number of firings were analyzed by the weighted mean difference (WMD), using random-effects model, and represented as risk difference with $95 \% \mathrm{CI}$. The $\mathrm{Q}$ test and $\mathrm{I}^{2}$ statistic were used to evaluate heterogeneity among studies. A Cochrane $\mathrm{Q}$ statistical $\mathrm{P}$ value $<0.10$ and/or $\mathrm{I}^{2}>50 \%$ was taken to indicate significant heterogeneity and both are represented within the forest plot figures. Publication bias was tested using funnel plots.

All statistical analyses were conducted using SPSS ${ }^{\circledR}$ version 22 software (SPSS, Inc., Chicago, IL) and $p$-values of $<0.05$ were considered statistically significant. 


\section{RESULTS}

\section{Study selection and characteristics}

The search strategy initially identified 51 studies. After exclusion of irrelevant studies, 10 potentially relevant were obtained for further assessment. Among these studies, 6 were excluded due to a lack of data.[6-11] Finally, 4 studies were included in our metaanalysis, with a total of 391 patients $($ LS group $=251$, RS group $=140)$.[12-15] The flow chart of the selection process is represented in Figure 1.

\section{Perioperative outcomes}

The results of the 4 studies included in this meta-analysis are summarized in Table 1. The first study showing the experience with the RS was published by Koh et al.[12] in 2014. Although there were a small number of patients included in the study, these preliminary results showed a trend towards improving results using the RS, with no AL in this group (compared to $10.5 \%$ in the LS group). Three years later, Guadagni et al.[13] showed a trend toward less firings and better postoperative outcomes in the RS group. Results were nonsignificant probably due to a small sample size.

Recently, a study published by our group confirmed these previous findings. The rectal transection was achieved in 1 or 2 firings in $91 \%$ of cases vs. $60 \%$ when using the LS ( $\mathrm{p}<0.001$ ), and the AL for the RS group was lower (4\% vs. 7\% in the LS group).[14]

Only one study found higher percentage of AL in the robotic group ( $>0.05)$, showing exactly the same number of firings regardless the stapler employed.[15]

Overall result of the four studies combined as presented in Figure 2 suggest a 56\% increased odds of AL for LS compared with RS; however, results are not statistically significant (OR: 1.56 ; 95\% CI: 0.59, 4.14). It also shows that the rectal stapling was achieved with less firings in the RS group [risk difference, (95\% CI): $0.42(-0.13,0.97)$ ], but the difference is not significant. (Fig. 3) 


\section{DISCUSSION}

The aim of this study was to investigate the impact of the RS on the rate of anastomotic leak. We have shown that the incidence of AL can be reduced by using the RS, although results are not statistically significant. To date, the RS has comparable outcomes to the LS[15] but seems to require fewer firings for a rectal section $(\mathrm{p}<0.05)$.[11,13]

The development of an anastomotic leak is multifactorial, and different variables have been widely investigated.[4,5] Variables related to intraoperative risk seem to be the most relevant. In particular the use of 3 or more firings, which have been shown to translate to lower rates of AL.[2,3] This is strongly related to technical difficulties, due to the limited space in the pelvis during a laparoscopic rectal transection. This is a challenging procedure, with limited traction manipulation.

The main advantage of the robotic stapler in this field is, undoubtedly, its $108^{\circ}$ of articulation in $\mathrm{x}$-axis and $54^{\circ}$ angulation in the $\mathrm{y}$-axis, which may provide more accurate firings in the confined space of the pelvis. Other technologic advances related to this type of stapler, such as the stable delivery of the staples, may have also influenced the outcomes. It is important to highlight that every study included in this meta-analysis has shown fewer number of stapler firings when using the robotic stapler, although results were not always statistically significant, probably due to a small sample size.

The only study that found slightly higher (but non-significant) percentage of AL in the robotic group was published in 2017 by Atasoy et al.[15] However, a selection bias could be influencing these results, as there were more male patients in the RS group with lower tumours. However, when patients are matched including gender and distance from the anorectal junction, Guadagni et al.[13] showed a trend toward less firings and better postoperative outcomes in the RS group.

This study has some limitations that deserve to be mentioned. The studies included did not mention the surgeons' expertise performing either laparoscopic or robotic anterior resection, which could be a bias regarding the learning curve using the staplers. The studies included in this analysis have a small sample size; however some of them have found statistically significant differences, which encourage performing a randomized trial with bigger sample size.

Also, comparison of anastomotic leakage rate between groups may have been influenced by several comorbidities, which are not addressed in some of these studies. 
However, our previous data published analysed in a multivariate analysis common factors that may influence the development of an anastomotic leak.

\section{CONCLUSIONS}

The main benefit of the robotic stapler is its maneuverability, simplifying the transection of the rectum in the pelvis. With the current literature evidence, the present study could not demonstrate a clear benefit of the robotic stapler over the laparoscopic stapler. Although the development of an anastomotic leak is multifactorial and unpredictable, our data may suggest that the use of the robotic stapler could help reducing the risk of anastomotic leak. There is a need of future randomized clinical trial in order to assess the possible benefits of the robotic stapler.

\section{COMPLIANCE WITH ETHICAL STANDARDS}

- No funding.

- Authors have no conflict of interest.

- All procedures performed in studies involving humans were in accordance with ethical standards of the institutional research committee and the 1964 Helsinki declaration and its later amendments.

- Informed consent was obtained from all participants.

\section{FIGURE LEGENDS}

Fig. 1. Flow chart of selection process

Fig. 2. Odds ratio in anastomotic leakage between robotic stapler (RS) and laparoscopic stapler (LS)

Fig 3. Risk difference in number of firings between robotic stapler (RS) and laparoscopic stapler (LS) 


\section{REFERENCES}

1. Jamali FR, Soweid AM, Dimassi H, Bailey C, Leroy J, Marescaux J (2008) Evaluating the degree of difficulty of laparoscopic colorectal surgery. Archives of surgery (Chicago, Ill : 1960) 143 (8):762-767; discussion 768. doi:10.1001/archsurg. 143.8 .762

2. Park JS, Choi GS, Kim SH, Kim HR, Kim NK, Lee KY, Kang SB, Kim JY, Lee KY, Kim BC, Bae BN, Son GM, Lee SI, Kang H (2013) Multicenter analysis of risk factors for anastomotic leakage after laparoscopic rectal cancer excision: the Korean laparoscopic

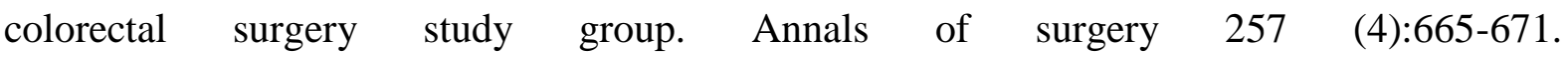
doi:10.1097/SLA.0b013e31827b8ed9

3. Braunschmid T, Hartig N, Baumann L, Dauser B, Herbst F (2017) Influence of multiple stapler firings used for rectal division on colorectal anastomotic leak rate. Surgical endoscopy 31 (12):5318-5326. doi:10.1007/s00464-017-5611-0

4. Qu H, Liu Y, Bi DS (2015) Clinical risk factors for anastomotic leakage after laparoscopic anterior resection for rectal cancer: a systematic review and meta-analysis. Surgical endoscopy 29 (12):3608-3617. doi:10.1007/s00464-015-4117-x

5. Kawada K, Sakai Y (2016) Preoperative, intraoperative and postoperative risk factors for anastomotic leakage after laparoscopic low anterior resection with double stapling technique anastomosis. World journal of gastroenterology 22 (25):5718-5727. doi:10.3748/wjg.v22.i25.5718

6. Priatno E, Kim SH (2015) Single stage robotic total mesorectal excision-a stepwise approach. Journal of visualized surgery 1:24. doi:10.3978/j.issn.2221-2965.2015.12.02

7. Leong QM, Son DN, Cho JS, Amar AH, Kim SH (2012) Robot-assisted low anterior resection for situs inversus totalis: a novel technical approach for an uncommon condition. Surgical laparoscopy, endoscopy \& percutaneous techniques 22 (2):e87-90. doi:10.1097/SLE.0b013e3182447ebc

8. Lightner AL, Kelley SR, Larson DW (2018) Robotic Platform for an IPAA. Diseases of the colon and rectum 61 (7):869-874. doi:10.1097/dcr.0000000000001125

9. Johnson CS, Kassir A, Marx DS, Soliman MK (2019) Performance of da Vinci Stapler during robotic-assisted right colectomy with intracorporeal anastomosis. Journal of robotic surgery 13 (1):115-119. doi:10.1007/s11701-018-0828-z

10. Ramos JR, Parra-Davila E (2014) Four-arm single docking full robotic surgery for low rectal cancer: technique standardization. Revista do Colegio Brasileiro de Cirurgioes 41 (3):216-223 
11. Holzmacher JL, Luka S, Aziz M, Amdur RL, Agarwal S, Obias V (2017) The Use of Robotic and Laparoscopic Surgical Stapling Devices During Minimally Invasive Colon and Rectal Surgery: A Comparison. Journal of laparoendoscopic \& advanced surgical techniques Part A 27 (2):151-155. doi:10.1089/lap.2016.0409

12. Koh FH, Tan KK, Lieske B, Tsang ML, Tsang CB, Koh DC (2014) Endowrist versus wrist: a case-controlled study comparing robotic versus hand-assisted laparoscopic surgery for rectal cancer. Surgical laparoscopy, endoscopy \& percutaneous techniques 24 (5):452456. doi:10.1097/SLE.0b013e318290158d

13. Guadagni S, Di Franco G, Gianardi D, Palmeri M, Ceccarelli C, Bianchini M, Furbetta N, Caprili G, D'Isidoro C, Moglia A, Melfi F, Buccianti P, Mosca F, Morelli L (2018) Control Comparison of the New EndoWrist and Traditional Laparoscopic Staplers for Anterior Rectal Resection with the Da Vinci Xi: A Case Study. Journal of laparoendoscopic \& advanced surgical techniques Part A. doi:10.1089/lap.2018.0218

14. Tejedor P, Sagias F, Nock D, Flashman K, Naqvi S, Kandala NL, Khan JS (2019) Advantages of using a robotic stapler in rectal cancer surgery. Journal of robotic surgery. doi:10.1007/s11701-019-00993-4

15. Atasoy D, Aytac E, Ozben V, Bayraktar O, Erenler Bayraktar I, Aghayeva A, Baca B, Hamzaoglu I, Karahasanoglu T (2018) Robotic Versus Laparoscopic Stapler Use for Rectal Transection in Robotic Surgery for Cancer. Journal of laparoendoscopic \& advanced surgical techniques Part A 28 (5):501-505. doi:10.1089/lap.2017.0545 
50 records identified through PUBMED searching
1 of additional records identified through Cochrane Database

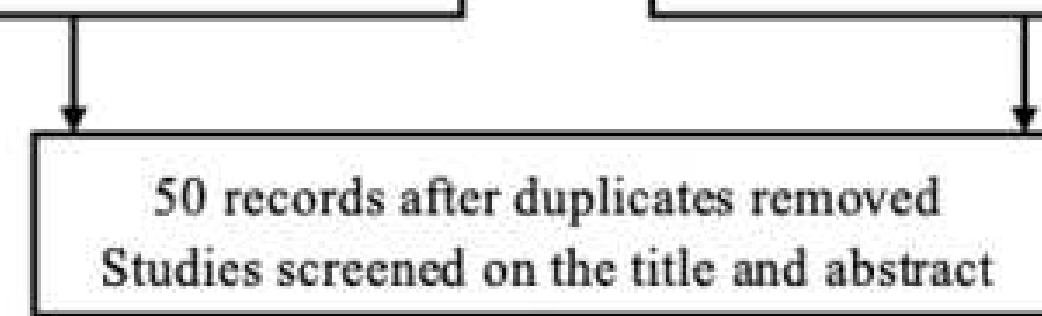

Studies screened on the title and abstract

导

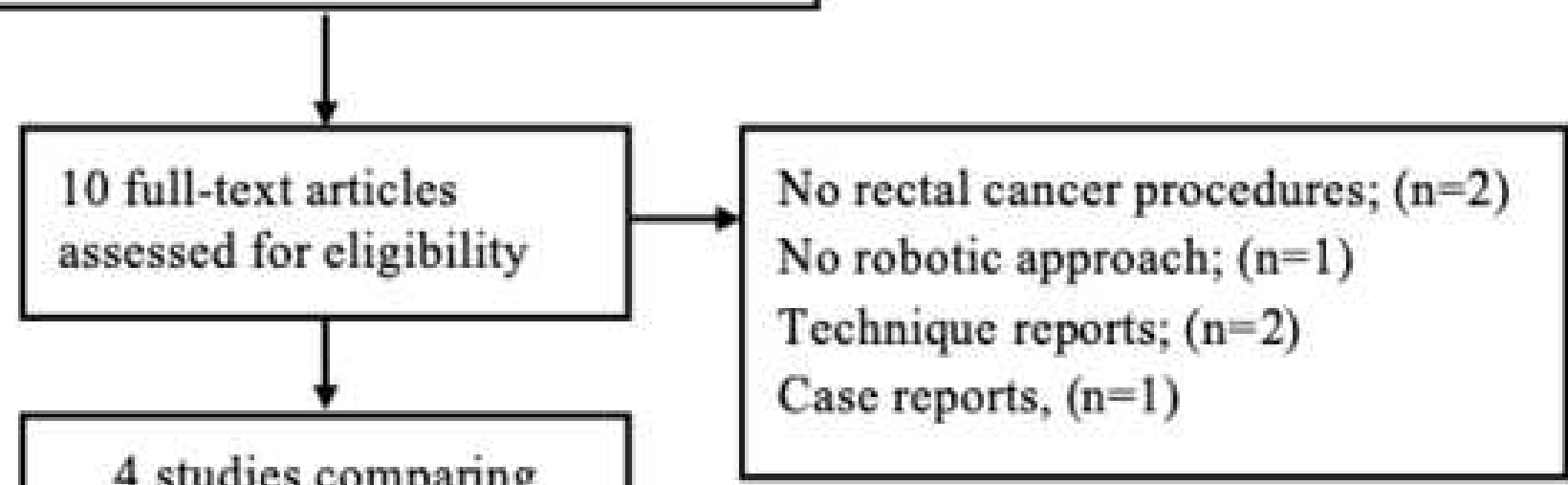

4 studies comparing robotic stapler with laparoscopic stapler

4 studies included in meta-analysis 

laparoscopic stapler (LS)

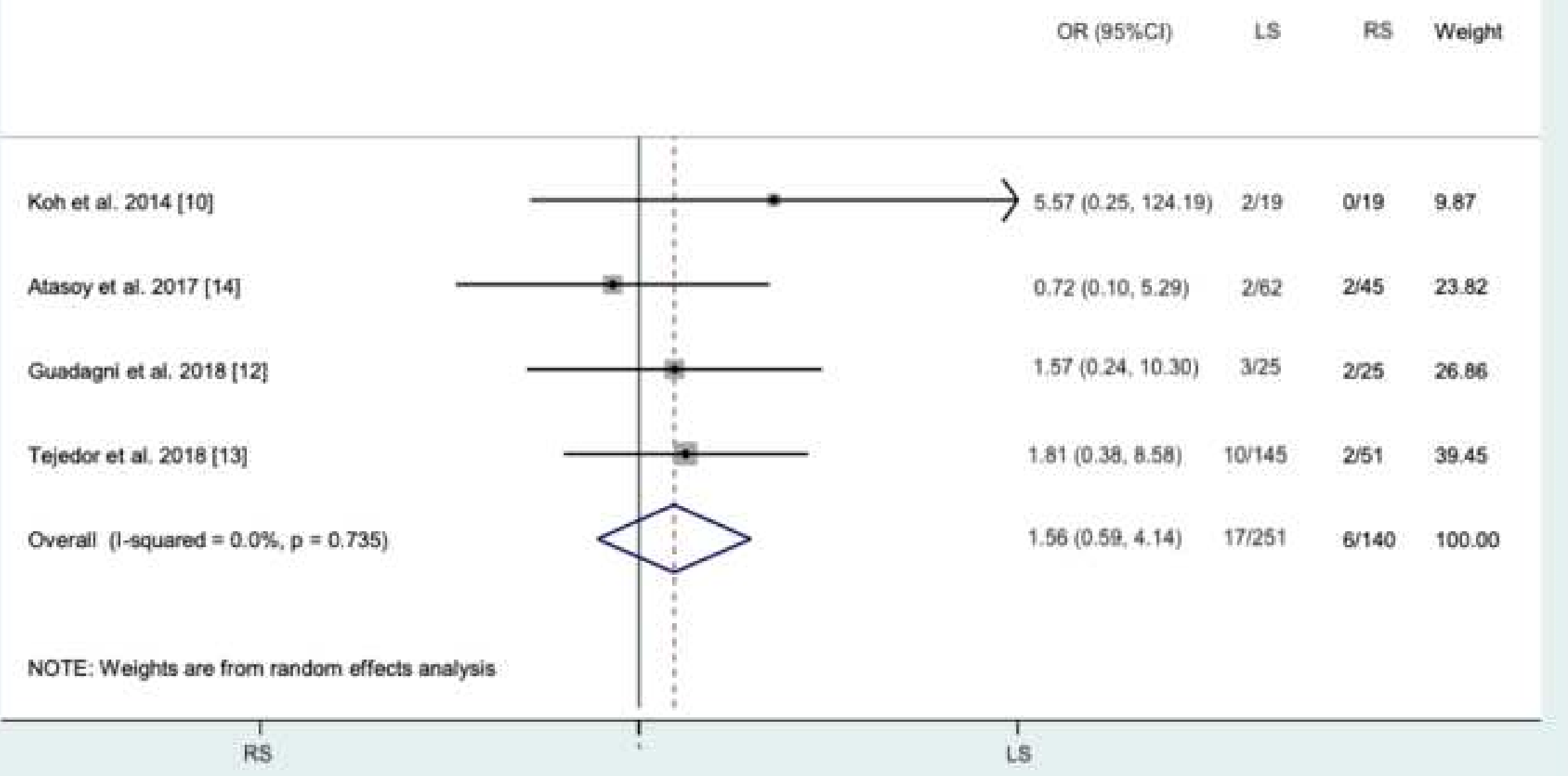




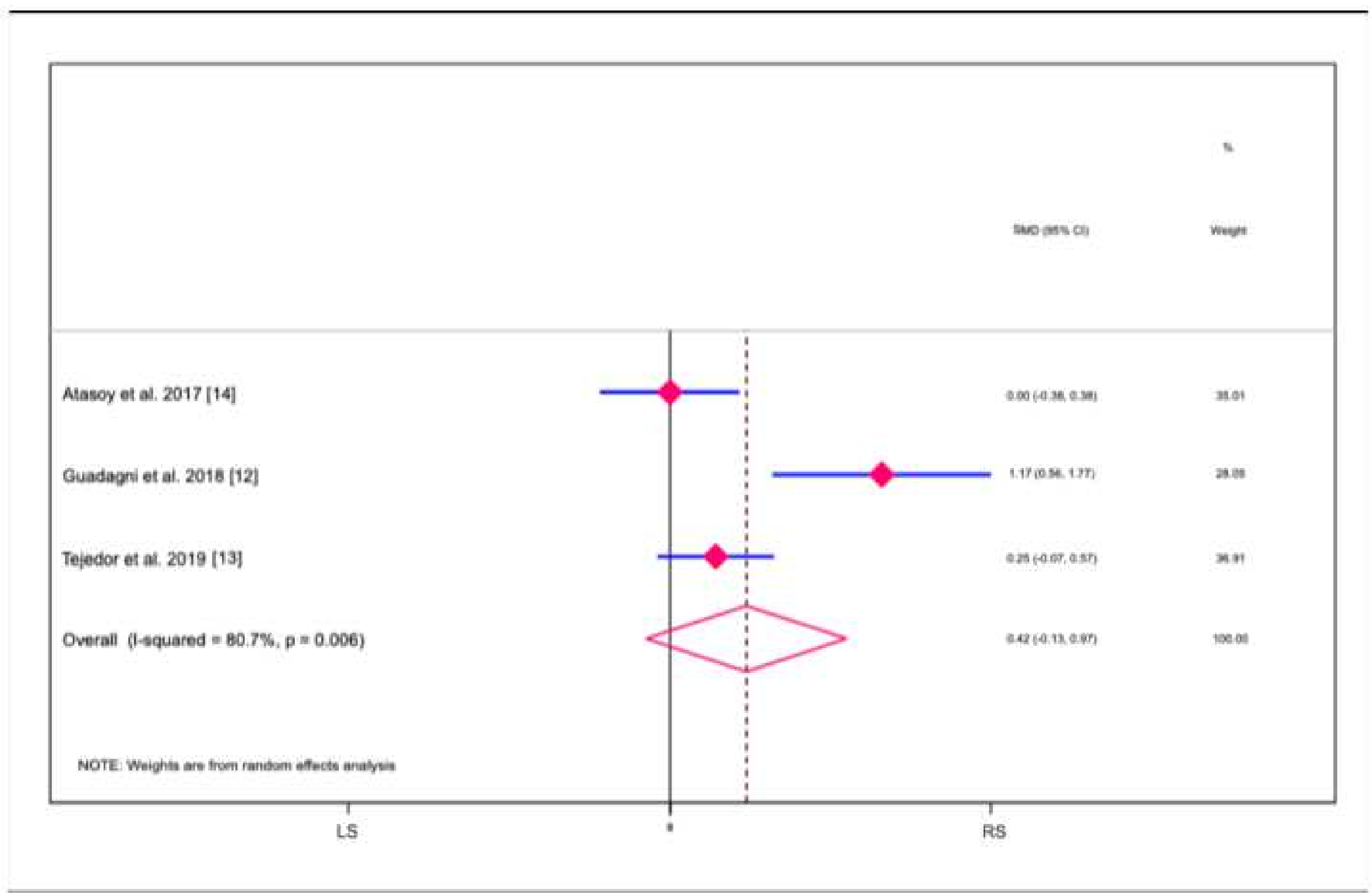

$\underline{\underline{t}}$ laparoscopic stapler (LS) 
Table 1. Baseline characteristics of the included studies regarding robotic stapler.

\begin{tabular}{|c|c|c|c|c|c|c|c|c|c|c|c|}
\hline & \multicolumn{2}{|c|}{$\begin{array}{l}\text { Number of } \\
\text { patients, } \mathrm{n}\end{array}$} & \multicolumn{2}{|c|}{$\begin{array}{c}\text { Number of firings, } \\
\text { mean (SD) }\end{array}$} & \multirow[t]{2}{*}{$\mathrm{p}$ value } & \multicolumn{2}{|c|}{$\mathrm{AL}, \mathrm{n}(\%)$} & \multirow[t]{2}{*}{$\begin{array}{c}\mathrm{p} \\
\text { value }\end{array}$} & \multicolumn{2}{|c|}{$\begin{array}{c}\text { Conversion } \\
\text { rate, } \%\end{array}$} & \multirow[t]{2}{*}{$\mathrm{p}$ value } \\
\hline & LS & RS & LS & $\mathrm{RS}$ & & LS & $\mathrm{RS}$ & & $\mathrm{LS}$ & RS & \\
\hline Atasoy et al. 2017[15] & 62 & 45 & $2(1)$ & $2(1)$ & NS & $2(3)$ & $2(4.4)$ & NS & 3 & 0 & NS \\
\hline Guadagni et al. 2018[13] & 25 & 25 & $2.7(0.7)$ & $2.1(0.2)$ & 0.0004 & $3(12)$ & $2(8)$ & NS & 0 & 0 & NS \\
\hline Koh et al. 2014[12] & 19 & 19 & - & & & $2(10.5)$ & 0 & NS & 5.3 & 10.5 & NS \\
\hline Tejedor et al. 2019[14] & 145 & 51 & $2.3(0.9)$ & $2.1(0.3)$ & 0.02 & $10(6.9)$ & $2(3.9)$ & NS & 0 & 0 & NS \\
\hline
\end{tabular}

AL, anastomotic leakage; LS, laparoscopic stapler; NS, non-significant; RS, robotic stapler; SD, standard deviation. 